\title{
Jejunal Stenosis
}

National Cancer Institute

\section{Source}

National Cancer Institute. Lejunal Stenosis. NCI Thesaurus. Code C78400.

Narrowing of the lumen of jejunum. 